\title{
Postos de Trabalho associados a Explosivos: principais Riscos e Fatores de Risco Laborais, Doenças Profissionais associadas e Medidas de Proteção recomendadas
}

www.rpso.pt/postos-de-trabalho-associados-a-explosivos-principais-riscos-e-fatores-de-risco-laborais-doencas-

profissionais-associadas-e-medidas-de-protecao-recomendadas/

January 25, 2017

WORKPLACES ASSOCIATED WITH EXPLOSIVES:

MAIN RISKS AND RISK FACTORS, ASSOCIATED PROFESSIONAL DISEASES AND

RECOMMENDED PROTECTIVE MEASURES

TIPO DE ARTIGO: Revisão Bibliográfica Integrativa AUTORES: Santos M , Almeida A .

RESUMO

Introdução/ enquadramento/ objetivos

Os profissionais envolvidos no manuseamento de explosivos estão sujeitos a diversos riscos/ fatores de risco; no entanto, a generalidade da bibliografia consultada aborda questões específicas e parcelares. Este estudo foi projetado para que seja possível ter uma perspetiva global que permita às equipas de Saúde Ocupacional avaliar e prevenir os riscos/ fatores de risco e doenças profissionais, bem como prescrever medidas de proteção (coletivas e individuais) capazes de preservar a saúde dos trabalhadores.

Metodologia

Trata-se de uma Revisão Bibliográfica Integrativa, iniciada através de uma pesquisa realizada em novembro de 2016 nas bases de dados "CINALH plus with full text, Medline with full text, Database of Abstracts of Reviews of Effects, Cochrane Central Register of Controlled Trials, Cochrane Database of Systematic Reviews, Cochrane Methodology Register, Nursing and Allied Health Collection: comprehensive, MedicLatina, Academic Search Complete e RCAAP".

Conteúdo

Os principais riscos/ fatores de risco associados a este setor são o ruido/ hipoacusia/ acufenos, explosão/ incêndio/ queimadura/ queda ou projeção de objetos/ fraturas/ contusão/ corte/ esmagamento, lesões oculares variadas, agentes químicos (dermatite, cancro, anemia), lesões músculo-esqueléticas, herniopatias, soterramento, vibrações, ultravioletas, desconforto térmico e stress. Emergiram como principais medidas de proteção individual a utilização de proteção auricular, calçado e farda adequados à condução de eletricidade eletrostática e máscara/ proteção respiratória.

Conclusões

Os principais riscos relacionam-se com a falta de proteção adequada para lidar com os efeitos da onda da explosão; no entanto, são também importantes os riscos químicos associados aos compostos envolvidos na fabricação dos explosivos e os riscos 
ergonómicos relacionados com o transporte manual de cargas.

Os artigos foram pouco completos em relação à descrição de eventuais medidas de proteção coletiva e individual, bem como na abordagem dada às doenças profissionais associadas. Para além disso, a maioria dos artigos publicados foi elaborada em contexto militar, pelo que as conclusões não poderão ser extrapoladas diretamente para o contexto laboral civil.

É necessário investigação relevante sobre o tema para aumentar a capacidade de tomada de decisão das equipas de saúde ocupacional.

PALAVRAS/ EXPRESSÕES- CHAVE: saúde ocupacional, saúde do trabalhador e medicina do trabalho; indústria de explosivos, acidentes causados por explosivos.

\section{ABSTRACT}

Introduction / objectives

The professionals involved in the handling of explosives are subject to several risks/ risk factors; however, the generality of the bibliography consulted addresses specific issues. This study was designed so that it is possible to have a global perspective that allows Occupational Health teams to assess and prevent occupational risk/ risk factors, as well as to prescribe protective measures (collective and individual), capable of preserving workers' health.

\section{Methodology}

This is an Integrative Bibliographic Review, initiated through a survey in November 2016 in the databases "CINALH plus with full text, Medline with full text, Database of Abstracts of Reviews of Effects, Cochrane Central Register of Controlled Trials, Cochrane Database of Systematic Reviews, Cochrane Methodology Register, Nursing and Allied Health Collection: comprehensive, MedicLatina, Academic Search Complete and RCAAP”.

Content

The main risks/ risk factors associated with this sector are noise/ hypoacusis/ tinnitus, explosion/ fire/ burn/ fall or projection of objects/ fractures/ contusion/ cut/ crush, various ocular lesions, chemical agents (dermatitis, cancer, anemia), musculoskeletal injuries, herniopathies, burial, vibrations, ultraviolet, thermal discomfort and stress. The use of ear protection, footwear and clothing appropriate to the conduction of electrostatic electricity and mask/ respiratory protection emerged as the main measures of individual protection.

Conclusions

The main risks are physical and relate to the lack of adequate protection to deal with the effects of the explosion wave; however, the chemical risks associated with the compounds involved in the manufacture of explosives and the ergonomic hazards related to the manual transport are also important.

The articles were incomplete in relation to the description of possible collective and individual protection measures, as well as in the approach given to associated occupational diseases. Moreover, most of the published articles were prepared in a military context, so the conclusions cannot be extrapolated directly into the civil context.

More relevant and available research is needed to increase the decision-making of occupational health teams.

KEY WORDS / EXPRESSIONS: occupational health, worker health; explosives industry, accidents caused by explosives. 
Os profissionais envolvidos no manuseamento de explosivos estão sujeitos a diversos riscos/ fatores de risco; para além disso, a generalidade da bibliografia consultada aborda riscos específicos e parcelares, não sendo fácil encontrar uma perspetiva global da área, a nível de Saúde Ocupacional. Justamente por isso, poderá existir alguma dificuldade na avaliação dos riscos/ fatores de risco, doenças profissionais e medidas de proteção (coletivas e individuais).

\section{METODOLOGIA}

Pergunta protocolar: Quais os principais riscos e fatores de risco existentes para os profissionais que manipulam Explosivos, eventuais doenças profissionais associadas e medidas de proteção recomendadas?

Em função da metodologia PICo, foram considerados:

-P (population): profissionais que manipulam Explosivos

-I (interest): reunir conhecimentos relevantes sobre os principais riscos e fatores de risco existentes para os profissionais que manipulam Explosivos, eventuais doenças profissionais associadas e medidas de proteção recomendadas, segundo os dados mais recentemente publicados

-C (context): saúde ocupacional nas empresas que utilizam explosivos

Foi realizada uma pesquisa em novembro de 2016 nas bases de dados "CINALH plus with full text, Medline with full text, Database of Abstracts of Reviews of Effects, Cochrane Central Register of Controlled Trials, Cochrane Database of Systematic Reviews, Cochrane Methodology Register, Nursing and Allied Health Collection: comprehensive, MedicLatina e Academic Search Complete". Utilizando a palavra-chave "explosives" foram obtidos 4288 artigos, com os critérios publicação igual ou superior a 2005 e acesso a texto completo; conjugando tal com as palavras/ expressões-chave "occupational, work, civil, accidents, diseases, risks e hazards”, obtiveram- se 15, 22, 8, 20, 70, 50 e 5 artigos; em função da língua original (portuguesa, inglesa ou espanhola) e da pertinência para o objetivo desta revisão, foram selecionados após a leitura do resumo e após a consulta do trabalho na íntegra, 3, 0, 1, 1, 0, 1 e 0 artigos. Paralelamente também se exploraram as expressões "explosive industry; explosive wastes; explosive substances, produts and materials; accidents caused by explosives e explosive agentes", tendo obtido 1, 0, 0, 0 e 63 trabalhos após a leitura do resumo; após o acesso ao artigo na íntegra manteve-se o interesse em 0, 0, 0, 0, e 3 artigos, respetivamente.

Contudo, como praticamente não se encontram estudos relativos à realidade portuguesa nestas bases de dados indexadas, os autores procuraram trabalhos inseridos no RCAAP (Repositório Científico de Acesso Aberto em Portugal). Aqui, utilizando as palavras-chave "explosivos", foram obtidos 361 documentos; vendo a interação destes com o termo "ocupacional" destacaram-se 21 trabalhos; após a leitura do resumo dos mesmos foram selecionadas duas investigações; após a consulta na íntegra perdeu-se o interesse em ambas.

O resumo da metodologia aplicada nesta revisão pode ser consultado nos fluxograma. A caraterização metodológica e resumo dos artigos selecionados está inserida no quadro 1. 
Ruído

Entre militares expostos frequentemente ao ruído associado às explosões, é mais prevalente a hipoacusia, quer subjetiva, quer quantificada com audiograma; tanto mais intensa quanto maior a proximidade da fonte do ruído e com a idade menos jovem; aliás a hipoacusia é a deficiência mais comum entre militares- ela é duas a cinco vezes superior nos miliares versus civis1, não sendo rara a coexistência de acufenos2,3, uma vez que o ruído súbito e intenso é muito mais lesivo que o ruído menos intenso, ainda que contínu01,2. Para além disso, o uso de proteção auricular poderá não ser sempre aconselhado, na medida em que diminui a perceção que o indivíduo tem em relação ao que o rodeia. A presença de agentes químicos ototóxicos poderá potenciar os danos2.

O trauma acústico, se muito intenso, poderá cursar com rutura do tímpano, alterações dos ossículos, membrana basilar e/ou de Reissner; podem também surgir a otalgia (dor no ouvido), otorragia (hemorragia do ouvido) e a vertigem3.

O trauma auditivo pode ser de primeiro grau (se afeta apenas as frequências próximas dos $4000 \mathrm{Hzs}$ ), de segundo grau (8000) e de $3^{\circ}$ grau (se também incluir o patamar dos 2000 $\mathrm{Hzs}) 3$.

Um estudo avaliou 73 indivíduos sujeitos a trauma secundário a explosão e concluiu que três a quatro meses após o evento se verificou a existência de uma perda neurosensorial de alta frequência em 78\%, mista em 19\% e perda de condução em 3\%; no geral, apenas os últimos apresentavam melhorias passado um ano3.

Alguns investigadores recomendam a distância mínima de segurança de oito metros do foco de explosão3.

\section{Alterações neurológicas}

Alguns estudos associados a militares expostos a explosões muito frequentes ao longo dos treinos, referem que estes apresentam uma prevalência superior de cefaleias, alterações do sono e da memória, sobretudo na proximidade das explosões4.

\section{Lesão ocular}

Nas explosões o segundo dano mais frequente são as lesões oculares (em primeiro lugar está a perfuração timpânica). As mais comuns são a abrasão da córnea, lacerações palpebrais, feridas abertas do globo ocular e a presença de corpos estranhos5.

As lesões secundárias a explosões neste contexto são categorizadas em quatro níveis de mecanismos: primário, secundário, terciário e quaternário. No primeiro caso a lesão é causada diretamente pela onda de explosão, ou seja, secundária ao efeito da pressurização e aquecimento do ar circundante à detonação, gerando uma pressão positiva de grande velocidade (10.000 a 30.000 metros por segundo), seguida de uma pressão negativa (que pode durar até dez vezes mais), que origina uma força de sucção do indivíduo e dos objetos circundantes, dependente da diferença de pressão e carga explosiva- daí que a distância seja uma fator importante para minimizar os danos. Para além disso, ambientes abertos permitem uma dissipação mais rápida e eficaz da onda. No segundo nível as lesões são geradas por corpos estranhos impulsionados pela onde de choque; são as mais frequentes ( 70 a $94 \%$ dos indivíduos com traumatismos por explosão). No terceiro nível o dano é secundário ao colapso estrutural dos edifícios circundantes. Por fim, na última categoria, incluem-se as queimaduras químicas e térmicas, inalação de agentes químicos tóxicos e exposição a radiações5.

A semiologia poderá abarcar a irritação ocular, dor, sensação de corpo estranho, 
alterações da acuidade, hemorragia e/ou edema periorbitário5.

Dever-se-á tentar inverter as pálpebras para identificar e remover eventuais corpos estranhos5.

Agentes químicos

-o exemplo do TNT (trinitrotolueno)

Esta substância é frequentemente utilizada como explosivo, dado ser económica e fácil de produzir; contudo, alguns autores consideram que esta pode aumentar o risco de surgirem hepatite, carcinoma hepático, anemia aplástica e hemolítica, bem como cataratas6,7. A hemólise (destruição das células sanguíneas) é predominantemente extravascular devido à retenção esplénica (no baço) dos eritrócitos alterados com a oxidação da hemoglobina (metemoglobina). Outras investigações também mencionam a ocorrência mais frequente de dermatite (irritação cutânea) e oligospermia (diminuição da concentração dos espermatozoides). As principais vias de absorção são a inalatória e a cutânea, pelo que a proteção respiratória isolada poderá não eliminar totalmente o risco, ainda que aprovada pela NIOSH. Os trabalhadores que utilizam este produto, para evitar a acumulação de eletricidade estática, deverão usar calçado condutor e farda de algodão, por exemplo7.

\section{Risco Oncológico}

Existem investigadores que associam o contato com alguns agentes químicos explosivos ao cancro da bexiga (ainda que a amostra tenha sido constituída por investigadores criminais que frequentemente contatavam com estas substâncias e outros agentes resultantes após combustão, originando hidrocarbonetos aromáticos policíclicos). Estes autores consideram que as principais vias de entrada destas substâncias eram a inalatória e a cutânea, ainda assim modulada pela ventilação, uso de EPI (equipamentos de proteção individual), hábito de ingerir sólidos e/ ou líquidos nos locais de trabalho e hidratação8,9.

Outros riscos/ fatores de risco não destacados na bibliografia consultada

Funcionários a laborar em empresas que utilizam explosivos apresentam também o fator de risco associado à queda/ projeção de objetos e eventuais fraturas, contusões, cortes e/ou esmagamento. Parte do equipamento e produtos utilizados têm de ser transportados manualmente, pelo que as cargas não são raras, bem como os eventuais riscos associados, como lesões músculo-esqueléticas e herniopatias (discais, inguinais, abdominais). Em alguns locais é possível que o funcionário fique soterrado ou inale poeiras. As viagens rodoviárias poderão ser prolongadas e/ou frequentes, o que implicará postura sentada mantida, ruido e vibrações. O trabalho ao ar livre poderá cursar com desconforto térmico (humidade, muito frio ou muito calor) e radiação ultravioleta. A eventual explosão e/ou incêndio poderão originar queimaduras em qualquer parte do corpo (e não apenas nos olhos, como alguns autores destacaram). Alguns trabalhadores poderão também ter de utilizar maquinaria produtora de ruido, vibrações e capaz de originar diversos tipos de acidentes. Por fim, alguns destes riscos poderão originar ansiedade no funcionário, o que, além de diminuir o bem-estar e qualidade de vida laboral e global, poderá implicar um maior risco de acidentes, secundário a decisões pouco ponderadas e impulsivas, moduladas pelo stress.

Normas orientadoras da ACT (Autoridade para as Condições do Trabalho)10

Em cada empresa deve existir apenas um elemento que assuma a responsabilidade global 
do trabalho com os explosivos (diretor da pedreira, contramestre ou até consultor). A formação teórica deste deve abarcar noções sobre cálculo e conceção dos tiros, vibrações, sopro da explosão e técnicas de tiros.

Os procedimentos de tiro devem estar afixados e divulgados; devem levar em conta a segurança dos trabalhadores, dos residentes e do ambiente. É necessário nomear e autorizar o carregador de fogo, seus ajudantes e os trabalhadores que armazenam estes produtos. O responsável deve confirmar a adequação e segurança dos produtos e timing dos tiros. Deve também ser delimitada uma zona de perigo, dentro da qual não deverá estar nenhum trabalhador. Os sistemas de alerta poderão incluir bandeiras, sons de aviso (para assinalar a evacuação e o fim da sessão de tiros); poderão também ser colocadas proteções. Os residentes deverão ser notificados direta e individualmente. Logo após o início dos tiros deve existir uma inspeção para detetar eventuais falhas. No final deve ser avaliada a possibilidade de existirem explosivos que não tenham sido acionados corretamente, de forma a evitar explosões acidentais e/ou roubos.

As chaves do armazém de explosivos devem permanecer em local seguro e de aceso restrito; devendo o material ser mobilizado apenas por funcionários habilitados e autorizados. O responsável pelo armazém deverá ainda garantir a segurança dos detonadores, registar todos os procedimentos, entregar e receber diretamente os produtos e informar imediatamente situações de perda ou roubo. Os detonadores devem estar em contentores com um sistema de encerramento sólido e cobertos por materiais antichoque e antiestáticos.

O nitrato de amoníaco deve ficar em local protegido mas corretamente ventilado. Para diminuir o risco de incêndio a zona circundante deve ser limpa a nível de vegetação e qualquer mancha de óleo ou outro produto devem ser removidos.

Os veículos utilizados no transporte devem ser mantidos limpos e devem ter número suficiente de extintores; é também necessário que se identifiquem com sinais, luzes ou bandeiras adequadas. Os reboques devem ter um sistema próprio de travagem ou ser acionado em caso de separação.

As ferramentas utilizadas para abrir os cartuchos ou buracos, bem como misturar explosivos devem ser fabricadas em materiais piróforos, como madeira, plástico antiestático ou metais não ferrosos.

Os camiões onde se faz a mistura de explosivos devem estar imobilizados num piso de terra, para dissipar as cargas eletrostáticas; a quantidade produzida deve ser a necessária apenas para o momento.

Cabe ao explorador do local nomear e autorizar os funcionários para executarem determinadas tarefas; para tal este deve realizar uma entrevista e efetuar um teste prático, para avaliar as capacidades.

As falhas detetadas deverão ser alvo de um inquérito para determinar e etiologia e atenuar a probabilidade de repetição.

Situações de má visibilidade (como nevoeiro, chuva ou neve) diminuem a segurança dos tiros.

Medidas de proteção coletiva

Para além das já mencionadas nas normas da ACT, poder-se-ão ainda considerar a rotatividade de tarefas, a colocação do menor número de funcionários nos locais e 
momentos mais lesivos, troca por agentes químicos menos tóxicos sempre que possível, boa manutenção e modernização dos equipamentos mecânicos, vigilância médica adequada e formação.

EPIs

Os EPIs mencionados nos artigos consultados restringem-se a proteção auricular, calçado e farda adequado à condução de eletricidade eletrostática e máscara/ proteção respiratória. Contudo, implicitamente, podem também ser necessários calçado adequado à eventual queda de objetos, farda de material não ígneo e adequada à temperatura, viseira, capacete, luvas (orientadas para a manipulação e cargas, agentes químicos, condução de eletricidade estática ou vibrações), cintas lombares, óculos (para a projeção de corpos estranhos, contato com poeiras/ agentes químicos e/ou com proteção ultravioleta) e creme de proteção solar cutânea.

Doenças Profissionais

Dentro dos trabalhos selecionados houve destaque para a hipoacusia, alguns cancros e dermatites. Contudo, em alguns casos poder-se-á também considerar tendinites/ tenossinovites e herniopatias variadas.

\section{CONCLUSÃO}

Os principais riscos são físicos e relacionam-se com a falta de proteção adequada para lidar com os efeitos da onda da explosão; no entanto, existem também riscos químicos associados aos compostos envolvidos no fabrico dos explosivos e riscos ergonómicos associados ao transporte manual de cargas.

Os artigos foram pouco completos em relação à descrição de eventuais medidas de proteção coletiva e individual, bem como na descrição das doenças profissionais associadas. Para além disso, a maioria dos artigos publicados foi elaborada em contexto militar, pelo que as conclusões não poderão ser extrapoladas diretamente para o contexto laboral civil.

A escassez de bibliografia publicada impede que a realidade portuguesa esteja retratada, pelo que seria pertinente motivar os profissionais da saúde ocupacional que trabalham no ramo (ou venham a trabalhar) para investigar aspetos relevantes do tema, divulgando as conclusões obtidas através da publicação de artigos

\section{CONFLITOS DE INTERESSE, QUESTÕES ÉTICAS E/OU LEGAIS}

Nada a declarar.

\section{AGRADECIMENTOS}

Nada a declarar.

\section{Bibliografia}

1)(sem autor) Severe Hearing Impairment among Military Veterans- United States, 2010. Morbidity and Mortality weekly report. 2011, 60(28), 955-958.

2)Wells T, Seelig A, Ryan M, Jones J, Hooper T, Jacobson I et al. Hearing loss associated with US Military combat deployment. 2015, 17(74), 34-42.

3)Arch-Tirado E, Garnica-Escamilla M, Delgado-Hernandez A, Campos- Munoz T, Rodrigues-Rodrigues L, Verduzco- Mendoza A. Trauma acústico generado por exposición a explosion de pólvora. Cirurgia Y Cirujanos. 2014, 82, 528-536. 
4)Carr W, Stone J, Waliko T, Young L, Snook T, Paggi M et al. Repeated low-level blast exposure: a descriptive human subjects study. Military Medicine. 2016, 181(5), 28-39.

5)Diaz D, Balparda K. Lesión ocular secundaria a trauma por explosión: lo que el médico de urgências debe saber. Archivos de Medicina de Urgência de México. 2013, 5(2), 70-77.

6)Naderi M, Ghanei M, Shohrati m, Saburi A, Baberei M, Najafian B. Systemic complication of trinitrotoluene (TNT) in exposed workers. Cutaneous and Ocular Toxicology. 2012, 32(1), 31-34.

7)Bradley M. 2,4,6- Trinitrotoluene (TNT) air concentrations, hemoglobin changes and anemia cases in respirator protected TNT munitions demilitarization workers. International Archives of Occupational Environmental Health. 2011, 84, 239-250.

8)Davis S, Tao X, Bernacki E, Alfriend A, Delowery M. Evaluation of a bladder cancer cluster in a population of criminal investigators with the bureau of alcohol, tobacco, firearms and explosives- part 2: the association of cancer risk and fire scene investigation. Journal of environmental and Public Health. 2013, 1-10.

9) Davis S, Tao X, Bernacki E, Alfriend A, Delowery M. Evaluation of a bladder cancer cluster in a population of criminal investigators with the bureau of alcohol, tobacco, firearms and explosives- part 1: the cancer incidence. Journal of Environmental and Public Health. 2013, 1-10.

10)Pina J, Gomes E, Carvalho M. Guia de preparação dos riscos relativos ao uso de explosivos. 2011, 1-15.

\section{Fluxograma}

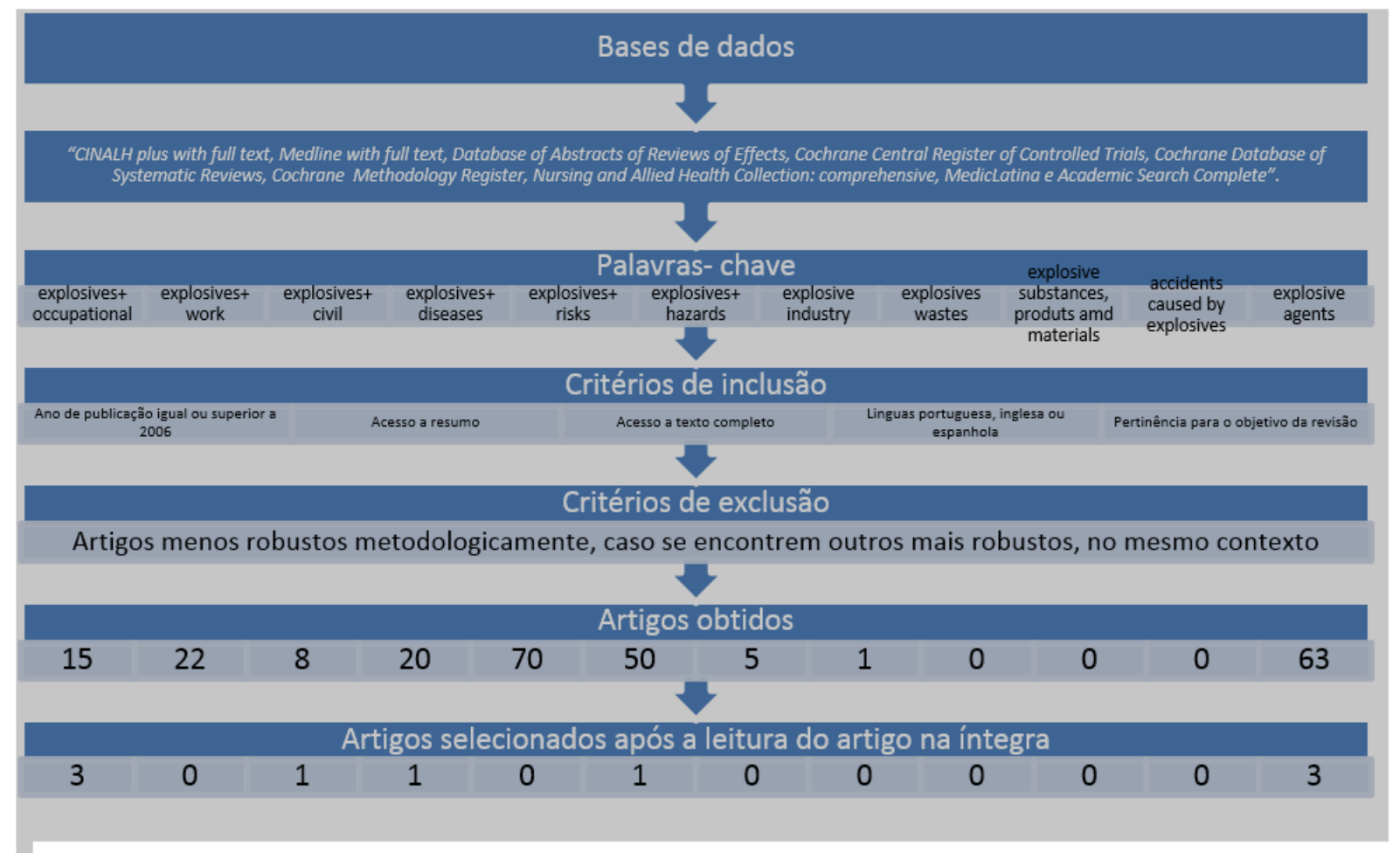

Quadro 1- Classificação metodológica dos artigos consultados 


\begin{tabular}{|c|c|c|}
\hline Artigo & Tipo de estudo & Resumo do estudo \\
\hline 1 & $\begin{array}{l}\text { Artigo de opinião/ } \\
\text { "Journal Club" }\end{array}$ & $\begin{array}{c}\text { O trabalho base destaca a hipoacusia em militares associada à } \\
\text { exposição ao ruído/ explosões. O autor também desenvolve } \\
\text { sumariamente a existência de EPls, prevalência da hipoacusia } \\
\text { entre militares e civis; realçando também que se trata de uma } \\
\text { patologia prevenível. }\end{array}$ \\
\hline 2 & $\begin{array}{l}\text { Observacional } \\
\text { Analítico de } \\
\text { Coorte }\end{array}$ & $\begin{array}{l}\text { Oobjetivo deste estudo foi quantificar o risco de hipoacusia entre } \\
\text { militares, em função dos seus eventos/ tarefas desempenhadas. } \\
\text { Os dados foram retirados de um estudo de coorte de quase } \\
49000 \text { militares. Concluiu-se que } 7,5 \% \text { apresentava hipoacusia } \\
\text { subjetiva, sendo que tal apresentou correlação com a hipoacusia } \\
\text { diagnosticada por audiograma. Esta também se demonstrou mais } \\
\text { prevalente nos militares presentes em combate, no sexo } \\
\text { masculino, em idades menos jovens e na maior proximidade à } \\
\text { explosão. }\end{array}$ \\
\hline 3 & Séries de Casos & $\begin{array}{l}\text { O objetivo deste trabalho foi descrever os danos audiologicos } \\
\text { associados a explosões. A amostra foi constituída por oito } \\
\text { indivíduos seguidos numa Unidade de Queimados e de } \\
\text { Reabilitação, expostos a explosão com pólvora. Cada um fez um } \\
\text { audiograma aéreo. Em todos se encontrou hipoacusia para todas } \\
\text { as frequências, bilateral, com intensidade a oscilar entre } \\
\text { moderada a intensa; o acufeno foi também muito prevalente. }\end{array}$ \\
\hline 4 & $\begin{array}{l}\text { Observacional } \\
\text { Analítico de } \\
\text { Coorte }\end{array}$ & $\begin{array}{l}\text { Neste trabalho os autores utilizaram uma amostra de militares } \\
\text { voluntários (5 instrutores, } 28 \text { formandos e } 7 \text { controlos), expostos } \\
\text { a treinos com explosões frequentes, de forma a avaliar eventuais } \\
\text { alterações neurológicas. Foram executados testes } \\
\text { neurocomportamentais, doseamentos sanguíneos, testes } \\
\text { vestibulares e exames de neuroimagem. }\end{array}$ \\
\hline 5 & $\begin{array}{l}\text { Revisão } \\
\text { Bibliográfica } \\
\text { Narrativa }\end{array}$ & $\begin{array}{l}\text { Os autores descrevem a epidemiologia das principais lesöes } \\
\text { oculares secundárias a explosão, respetiva categorização, } \\
\text { fisiopatologia, semiologia e orientação terapêutica para médicos } \\
\text { generalistas a exercer em urgências. }\end{array}$ \\
\hline 6 & $\begin{array}{l}\text { Observacional } \\
\text { Analítico } \\
\text { Transversal }\end{array}$ & $\begin{array}{l}\text { O realce neste trabalho é dado ao uso de TNT como explosivo e } \\
\text { consequências médicas associadas, nomeadamente a nível de } \\
\text { hepatite, anemia, cataratas a patologia oncológica. }\end{array}$ \\
\hline 7 & $\begin{array}{l}\text { Observacional } \\
\text { Analítico de } \\
\text { Coorte } \\
\text { Retrospetivo }\end{array}$ & $\begin{array}{l}\text { Também aquí se destacou o uso de TNT como explosivo e } \\
\text { eventuais consequências para a saúde, salientando-se as } \\
\text { anemias aplástica e hemolítica; os autores mencionaram também } \\
\text { as principais vias de absorção e EPls. Após uma exposição de } \\
\text { sete meses foi doseada a concentração de hemoglobina. Os } \\
\text { autores concluíram que a proteção respiratória poderá não ser } \\
\text { suficiente para atenuar o risco de anemia. }\end{array}$ \\
\hline 8 & $\begin{array}{l}\text { Observacional } \\
\text { Analítico de Caso- } \\
\text { Controlo }\end{array}$ & $\begin{array}{l}\text { Este documento avaliou a associação entre o cancro de bexiga e } \\
\text { a presença em investigações criminais em ambientes com } \\
\text { agentes químicos explosivos e hidrocarbonetos aromáticos } \\
\text { policíclicos. Encontrou-se uma relação estatisticamente } \\
\text { significativa com uma razão de riscos de } 19 \text {. A amostra global foi } \\
\text { constituída por quase } 4000 \text { trabalhadores; os controlos foram os } \\
\text { funcionários da instituição não expostos a estes agentes. Os } \\
\text { autores concluíram que as principais vias de exposição eram a } \\
\text { inalatória e a cutânea. O risco é modulado também pela } \\
\text { ventilação, uso de EPls e ingestão de comida e bebida nas cenas } \\
\text { de crime. }\end{array}$ \\
\hline 9 & $\begin{array}{l}\text { Observacional } \\
\text { Analítico de } \\
\text { Coorte }\end{array}$ & $\begin{array}{l}\text { Este artigo foi elaborado em conjunto com o anterior e retrata o } \\
\text { seguimento efetuado entre } 1993 \text { e } 2007 \text {, relativamente ao cancro } \\
\text { de bexiga nos profissionais mencionados. }\end{array}$ \\
\hline
\end{tabular}

Santos M, Almeida A. Postos de Trabalho associados a Explosivos: principais Riscos e Fatores de Risco Laborais, Doenças Profissionais associadas e Medidas de Proteção recomendadas. Revista Portuguesa de Saúde Ocupacional. 2017, volume 3, 1-7. 